\title{
A Novel Generalized Nonholonomy Criteria and Physical Interpretation of Holonomic/Nonholonomic Constraints of a Free-Flying Space Robot with/without Interaction with a Flying Target Satellite
}

\author{
Murad Shibli ${ }^{1}$, Sohail Anwar ${ }^{2}$ \\ ${ }^{1}$ College Requirement Unit, Abu Dhabi Polytechnic, Institute of Applied Technology, \\ Abu Dhabi, United Arab Emirates \\ ${ }^{2}$ Altoona College, Penn State University, Altoona, USA \\ E-mail:murad.alshibli@iat.ac.ae
}

Received December 15, 2010; revised June 29, 2011; accepted July 5, 2011

\begin{abstract}
This paper presents a new nonholonomy criteria and reveals the physical interpretation of holonomoic and nonholonomic constraints acting on a free-flying space robot with or without interaction with a free Flying/Floating target object. The analysis in this paper interprets the physical interpretation behind such constraints, and clarifies geometric and kinematic conditions that generate such constraints. Moreover, a new criterion of finding the holonomy/nonholonomy of constraints impose on a free-flying space robot with or without interaction with a floating object is presented as well. The proposed criteria are applicable in case of zero or non-zero initial momentum conditions. Such nonholonomy criteria are proposed by utilizing the concept of orthogonal projection matrices and singular value decomposition (SVD). Using this methodology will also enable us to verify online whether the constraints are violated in case of real-time applications and to take a correction action or switch the controllers. This criterion is still yet valid even the interaction with floating object is lost. Applications of the proposed criteria can be dedicated to in-orbit servicing robotic satellite to capture malfunctioned spacecrafts and satellites, docking space of NASA and Russian shuttles with International Space Station (ISA), building in-orbit stations, space rescue missions and asteroids dust sampling. Finally, simulation results are presented to demonstrate the effectiveness of the proposed criterion.
\end{abstract}

Keywords: Honlonomic and Nonholonomic Constraints, Nonholonomy, Free-Flying Space Robot, Target Satellite

\section{Introduction}

The most complex space application of robotics is inorbit servicing. The scientific and commercial motive for robotic spacecraft and their future central role in space activities is comparatively new, particularly for general earth orbit operations which are presently dominated by manned missions. Particularly, the purpose of a dedicated robotic satellite for in-orbit servicing is to capture malfunctioned spacecrafts and satellites and perform maintenance and services to effectively increase the overall reliability of all accessible space systems. Servicing satellite equipped with robot arms can be employed for recovering the attitude, charging the exhaust- ing batteries, attaching new thrusters, and replacing the failed parts like gyros, solar panels or antennas of another satellite. There is no doubt that robotic and autonomous systems in space will contribute considerably to the future commercialization of space industry, saving billions of commercial space missions, extending their servicing age and making the space less polluted.

Many techniques of kinematics and dynamic modeling of space robots have been developed in $[1-5,10,11]$. Kinematics and dynamics motion of a space robot system are developed based on the concept of a Virtual Manipulator (VM) [2,3]. Another modeling of kinematics of a free-flying space robot is proposed by deriving the total momenta without the need of a pre-selected body [4]. 
While in [5] the kinematics of a free-flying multi-body system is investigated by introducing the conservation of momentum and deriving a new Jacobian matrix called the Generalized Jacobian. In research [6], the kinematics and dynamics of free-floating coordinated space robotic system with closed kinematic constraints are developed. An approach to position and force control of free-floating coordinated space robots with closed kinematic constraints is proposed for the first time. Unlike previous coordinated space robot control methods which are for open kinematic chains, the method presented here addresses the main difficult problem of control of closed kinematic chains. The controller consists of two parts, position controller and internal force controller, which regulate, respectively, the object position and internal forces between the object and end-effectors. A planar FFSR with a 2 DOFs manipulator is selected to test the algorithm and simulation results illustrate that the path following is realized precisely. The genetic algorithm with wavelet approximation is applied to nonholonomic motion planning in [7]. The problem of nonholonomic motion planning is formulated as an optimal control problem for a drift.

When a robot end-effecter interacts with a stationary environment or moving object, it imposes a geometric holonomic (integrable) constraint $[2,9,11]$. The conservation of momentum exerts kinematic-like constraints on a space robot in the absence of external forces. The linear momentum is considered as holonomic but the angular momentum as nonholonomic (non-integrable) [11]. Control of nonholonomic system received a great attention of the research developed in $[8,11-13,14]$. Research [15] addresses modeling, simulation and controls of a robotic servicing system for the hubble space telescope servicing missions. The simulation models of the robotic system include flexible body dynamics, control systems and geometric models of the contacting bodies. These models are incorporated into MDA's simulation facilities, the multibody dynamics simulator "space station portable operations training simulator (SPOTS)". In [16], the kinematics of the FFSR is introduced firstly. Then the null space approach is used to reparameterize the path: the direction and magnitude are decoupled and no direction error is introduced. And the Newton iterative method is adopted to find the optimal magnitude of the joint velocity. The inverse kinematic control based on mutual mapping neural network of free-floating dual-arm space robot system without the basepsilas control is discussed in [17]. With the geometrical relation and the linear, angular momentum conservation of the system, the generalized Jacobian matrix is obtained.

To solve the challenge of nonintegrability of principle of conservation of angular momentum many researchers have proposed different schemes. A nonholonomic path planning of space robots is proposed in [8] via bi-directional approach. The spacecraft orientation can be controlled in addition to the joint variables of the manipulator, by actuating only the joint variables, if the trajectory is carefully planned. A major characteristic of a space robot is clearly the distinction from ground-based robot is the lack of a fixed base in space environment [5]. Since the conservation of momentum exerts kinematiclike constraints on a space robotic system in the absence of external forces, one may raise the question: what is the physical interpretation of such a behavior? Some researchers have looked at this problem from trajectory planning point of view, From trajectory planning point of view, not all trajectories and displacements (velocities) are allowed due to the conservation of momentum and geometric constraints $[8,11]$. The physical meaning behind these constraints is that they restrict the kinematically possible displacements (possible values of the velocities) of the individual parts of the system.

The physical characteristics of the nonholonomic constraints are exhibited by the fact that even if the manipulator joints return to their initial configuration after a sequence of motion, the vehicle orientation may not be the same as the initial value [8]. If a space robot is operated in a certain task, position and attitude of the base satellite are disturbed by reaction forces and moments due to the robot motion, so it cannot accomplish a task without provision for this disturbance. No space manipulator can avoid the reaction disturbance. Physical interpretation of such behavior will give us more idea about the nature of holonomic and nonholonomic constraints and geometric conditions that generate those constraints. Verifying the intergrability of holonomic and nonholnomic constrained systems has attracted the attention of several studies [8-18]. Frobenius theorem is a wellknown approach to answer the question of integrability of such systems under concern. Conditions of the integrability of nonholonomic systems are reported using Lie algebra techniques in [8]. A necessary and sufficient condition is reported by using what is called bilinear covariants in [12,13-18]. Nonholomic behavior of a freeflying space robot is investigated in the absence of external forces by Lie algebra techniques [8]. Differential-form-based integrability conditions for dynamic constraints using the Frobenius theorem are proposed in [19]. In the latter, the conditions can be used for the classification of holonomic and nonholonomic constraints. Unfortunately, using Lie algebra and bilinear covariant is cumbersome and time consuming in the case of complicated space robotic systems.

This paper presents a new methodology to determine holonomy/nonholonomy of constraints impose on a free- 
flying space robot with or without interaction with a floating object. In this work a physical interpretation of nonholnomic constraints is presented. It gives an insight of nonholonomic constraints and provides more information of a space robot behavior, especially in control which is more difficult than conventional holonomic systems. The holonomy criterion is proposed by utilizing the concept of orthogonal projection matrices and singular value decomposition (SVD). This criteria is economic (from computational view point) can easily be used to verify the holonomy of a space robot exposed to different types of constraints. Using this methodology will also enable us to verify online whether the constraints or their initial conditions are violated in case of real-time applications and to take a corrective action or switch the controllers if needed. Such a physical interpretation will provide us with a better understanding of a space robot especially in contact task planning and control, which are more difficult than conventional holonomic systems.

The paper is organized as follows: In Section 2, modeling of kinematics, linear and angular momentum are derived. In Section 3, a physical interpretation of nonholnomic constraints is presented. In Section 4, nonholonomy criteria in case of zero initial momentum conditions, meanwhile in Section 5 a nonholonomy criteria of non-zero initial momentum conditions space robotic system is presented. Finally, simulation results and conclusions are presented respectively in Sections 6 and 7 to demonstrate the analytical results.

\section{Kinematics and Momentum Modeling}

\subsection{Nomenclature}

All generalized coordinates are measured in the inertial frame unless another frame is mentioned as follows:

$m_{i}$ : the mass of the $i$ th body;

$I_{i} \in R^{3}:$ the inertia of the $i$ th body;

$q \in R^{n}:$ the robot joint variable vector $q\left(q_{1}, q_{2}, \cdots, q_{n}\right)^{\mathrm{T}}$;

$R_{b} \in R^{3}$ : the position vector of the centroid of the base;

$R_{T} \in R^{3}:$ the position vector of the target satellite;

$r_{i} \in R^{3}$ : the position vector of the $i$ th joint;

$R_{T / E E} \in R^{3}$ : the position vector of the target satellite centroid with respect to the end-effecter (EE);

$V_{b} \in R^{3}:$ the linear velocity of the base;

$\Omega_{b} \in R^{3}$ : the base angular velocity vector;

$U_{3}$ : the $3 \times 3$ identity matrix.

\subsection{Kinematics}

The purpose of this part is to model the kinematics of a free-flying space robotic manipulator in contact with a captured satellite as a whole. In this model the contact between the space robot and the target satellite is assumed established and not escaped.

Our combined system can be modeled as a multi-body chain system composed of $n+2$ rigid bodies. While the manipulator links are numbered from 1 to $n$, the base satellite (body 0 ) is denoted by $b$, in particular, and the $(n+1)$ th body (the target satellite) by $T$. Moreover, This multi-body system is connected by $n+1$ joints, which are given numbers from 1 to $n+1$. Where the end-effecter is represented as the $(n+1)$ th joint as shown in Figure 1.

We assume that all system bodies are rigid, the contact surfaces are frictionless and known. Also the effect of gravity gradient, solar radiation and aerodynamic forces are weak and neglected. It is assumed also that the base satellite is reaction-wheel actuated.

Referring to Figure 1, the position vector of the ith body centroid with respect to the inertial frame can be expressed as [20-22]

$$
R_{i}=R_{b}+R_{i / b}
$$

where the relative vector $R_{i / b}$ is the position of the $i$ th body centroid with respect to the base frame.

Upon differentiating both sides of (1) with respect to time, the relationship between the ith body velocity

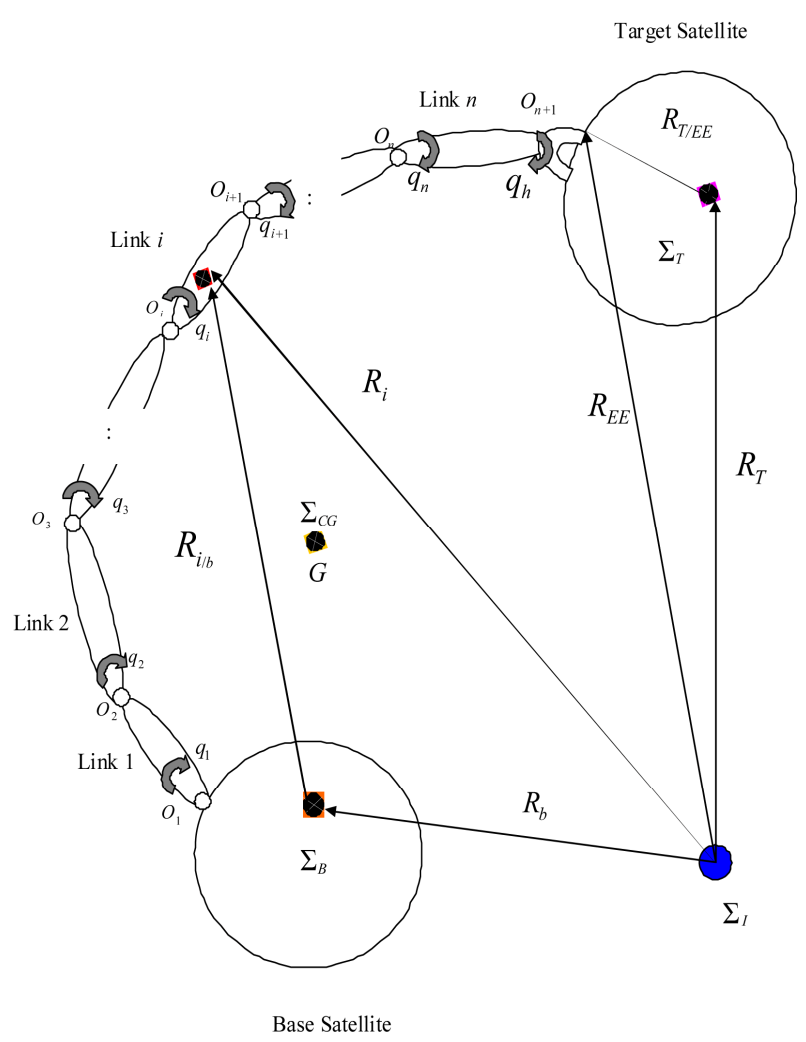

Figure 1. Multi-body diagram of a free-floating space robot in contact with a target satellite. 


$$
V_{i}=V_{b}+\Omega_{b} \times R_{i / b}+v_{i}
$$

where $v_{i}$ is the linear velocities of the ith body in base coordinates. Now in the case of any ith body of the manipulator, the velocity $v_{i}$ can be expressed in terms of the linear Jacobian matrix as

$$
v_{i}=J_{L_{i}} \dot{q}
$$

where

$$
J_{L_{i}}=\left[z_{1} \times\left(R_{i}-r_{1}\right), z_{2} \times\left(R_{i}-r_{2}\right), \cdots, z_{i} \times\left(R_{i}-r_{i}\right), 0, \cdots, 0\right]
$$

The end-effecter tip velocity is given by

$$
V_{E E}=V_{b}+\Omega_{b} \times R_{E E / b}+J_{L_{E E}} \dot{q}
$$

Additionally, the velocity $V_{T}$ of the target satellite in the reference frame can be obtained by deriving Equation applying (1) as

$$
V_{T}=V_{b}+\Omega_{b} \times R_{T / b}+J_{L_{T}} \dot{q}+\omega_{T} \times R_{T / E E}+v_{T}
$$

Since the target satellite is not stationary, (6) shows the relative linear and angular velocities $v_{T}, \omega_{T}$ between the end effecter and the target satellite and measured in the base frame.

Another relationship is needed between the ith body angular velocity $\Omega_{i}$ and joint angular velocity

$$
\Omega_{i}=\Omega_{b}+\omega_{i}
$$

where $\omega_{i}$ is the angular velocities of the $i$ th body in base coordinates and $\omega_{i}$ in case of the manipulator is given by

$$
\omega_{i}=J_{A_{i}} \dot{q}
$$

where the angular Jacobian

$$
J_{A_{i}}=\left[z_{1}, z_{2}, \cdots, z_{i}, 0, \cdots, 0\right]
$$

While in the case of the target satellite, the absolute angular velocity of can be expressed as

$$
\Omega_{T}=\Omega_{b}+J_{A_{E E}} \dot{q}+\omega_{T}
$$

\subsection{Linear and Angular Momentum}

The linear and angular momentum of a multi-body system is a key part in understanding the motion of the system when it is not subjected to external forces. They may impose kinematic-like constraints when the system is free of any external force.

The linear $P$ momentum and angular momentum $L$ of the whole system is given by

$$
P \equiv \sum_{i=0}^{n+1} m_{i} V_{i}
$$

$$
L \equiv \sum_{i=0}^{n+1}\left({ }^{B} I_{i} \Omega_{i}+m_{i} R_{i} \times V_{i}\right)
$$

By means of (1)-(10), linear and angular momentum in (11)-(12) can then be represented in a compact form as

$$
\begin{aligned}
{\left[\begin{array}{c}
P \\
L
\end{array}\right]=} & {\left[\begin{array}{cc}
M_{V_{b}} & M_{V_{b} \Omega_{b}} \\
M_{V_{b} \Omega_{b}}^{T} & M_{\Omega_{b}}
\end{array}\right]\left[\begin{array}{c}
V_{b} \\
\Omega_{b}
\end{array}\right]+\left[\begin{array}{c}
M_{V_{b} q} \\
M_{\Omega_{b} q}
\end{array}\right] \dot{q} } \\
& +\left[\begin{array}{ll}
M_{V_{b} \omega_{T}} & M_{V_{b} v_{T}} \\
M_{\Omega_{b} \omega_{T}} & M_{\Omega_{b} v_{T}}
\end{array}\right]\left[\begin{array}{c}
\omega_{T} \\
v_{T}
\end{array}\right]
\end{aligned}
$$

where each block of the matrix is defined as follows

$$
\begin{gathered}
M_{V_{b}} \equiv U_{3} \sum_{i=0}^{n+1} m_{i} \in R^{3 \times 3} \\
M_{V_{b} \Omega_{b}} \equiv-\sum_{i=0, i \neq b}^{n+1} m_{i}\left[R_{i / b} \times\right] \in R^{3 \times 3} \\
M_{V_{b} q} \equiv \sum_{i=0, i \neq b}^{n+1} m_{i} J_{L_{i}} \in R^{3 \times n} \\
M_{\Omega_{b}} \equiv \sum_{i=0, i \neq b}^{n+1}\left\{I_{i}+m_{i} D\left(R_{i / b}\right)\right\}+I_{b} \in R^{3 \times 3} \\
M_{\Omega_{b} q} \equiv \sum_{i=0, i \neq b}^{n+1}\left\{{ }^{B} I_{i} J_{A_{i}}+m_{i}\left[R_{i / b} \times\right] J J_{L_{i}}\right\} \in R^{3 \times n} \\
M_{V_{b} \omega_{T}} \equiv-m_{n+1}\left[R_{T / E E} \times\right] \in R^{3 \times 3} \\
M_{\Omega_{b} \omega_{T}} \equiv m_{i} D\left(R_{T / E E}\right)+{ }^{b} I_{n+1} \in R^{3 \times 3} \\
M_{V_{b} v_{T}} \equiv U_{3} m_{n+1} \in R^{3 \times 3} \\
M_{\Omega_{b} v_{T}} \equiv-m_{n+1}\left[R_{n+1} \times\right] \in R^{3 \times 3}
\end{gathered}
$$

Note that the matrix function $[R \times]$ for a vector $R=\left[R_{x}, R_{y}, R_{z}\right]^{T}$ is defined as

$$
[R \times] \equiv\left[\begin{array}{ccc}
0 & -R_{z} & R_{y} \\
R_{z} & 0 & -R_{x} \\
-R_{y} & R_{x} & 0
\end{array}\right] \in R^{3 \times 3}
$$

and

$$
\begin{aligned}
D(R) & \equiv[R \times]^{T}[R \times] \\
& =\left[\begin{array}{ccc}
R_{y}^{2}+R_{z}^{2} & -R_{x} R_{y} & -R_{x} R_{z} \\
-R_{x} R_{y} & R_{x}^{2}+R_{z}^{2} & -R_{y} R_{z} \\
-R_{x} R_{z} & -R_{y} R_{z} & R_{x}^{2}+R_{y}^{2}
\end{array}\right] \in R^{3 \times 3}
\end{aligned}
$$

and the sub-matrices of the Jacobian of the ith body representing the linear and angular parts are defined before.

Note that as in (13) the system is subjected to a nonholonomic (non-integrable) constraint because of conservation of angular momentum in the absence of external forces. On the contrast, the linear momentum results 
in a holonomic (integrable) constraint.

\section{Physical Interpretation of Nonholonomic Constraints}

\subsection{Free-Flying Space Robot}

In this section a physical explanation of the nonholonomic constraint imposed on a free-flying space robot. When no external forces are applied, and in the absence of gravity and dissipation forces, the linear and angular momentum of the multibody system are conserved.

Then by virtue of the principle of virtual work by d'Almberts-Lagrange equation [12,18]

$$
\sum_{i=0}^{n}\left(m_{i} \ddot{R}_{i}-f_{i}-F_{i}\right) \delta R_{i}=0
$$

In which it implies no work as a result of the virtual displacement $\delta R_{i}$ measured in frame fixed at the center of mass as shown in Figure 1. The position vector of the $i$ th body can be given as

$$
R_{i}=R_{b}+R_{i / b}
$$

where $R_{i}$ is the position vector from the fixed center $C$ to the $i$ th body, $R_{b}$ is the position vector form the center of mass to the base satellite, and $R_{i / b}$ is the vector from the base to ith body.

Now taking the displacement of vector $R_{i}$, we obtain

$$
\mathrm{d} R_{i}=\mathrm{d} R_{b}+\mathrm{d} \phi \times R_{i / b}+J_{L i} \mathrm{~d} q_{i}
$$

Similarly, the virtual displacement can be stated as

$$
\delta R_{i}=\delta R_{b}+\delta \phi \times R_{i / b}+J_{L i} \delta q_{i}
$$

where $\delta \phi$ is the angular virtual displacement by which the base body rotates about the virtual axis of rotation $\mathrm{Z}, \delta q_{i}$ is the virtual angular displacement of robot $i$ th body, and $J_{L i}$ is the linear Jacobian defined as

$$
\sum_{i=o}^{n} k_{i} \times\left(R_{i}-r_{i}\right) \text {. }
$$

Substituting $\sum_{i=o}^{n} k_{i} \times\left(R_{i}-r_{i}\right)$ for $J_{L i}$ in Equation (28)

$$
\delta R_{i}=\delta R_{b}+\delta \phi \times R_{i / b}+\sum_{i=o}^{n} k_{i} \times\left(R_{i}-r_{i}\right) \delta q_{i}
$$

Now substitute the virtual displacement (29) into d'Almberts-Lagrange Equation (25) to have

$$
\begin{aligned}
& m_{i} \ddot{R}_{i} \cdot \delta R_{b}+m_{i} \ddot{R}_{i} \cdot \delta \phi \times R_{i / b}+m_{i} \ddot{R}_{i} \cdot \sum_{i=o}^{n} k_{i} \times\left(R_{i}-r_{i}\right) \delta q_{i} \\
& =f_{i} \cdot \delta R_{b}+f_{i} \cdot \delta \phi \times R_{i / b}+f_{i} \cdot \sum_{i=o}^{n} k_{i} \times\left(R_{i}-r_{i}\right) \delta q_{i} \\
& \quad+F_{i} \cdot \delta R_{b}+F_{i} \cdot \delta \phi \times R_{i / b}+F_{i} \cdot \sum_{i=o}^{n} k_{i} \times\left(R_{i}-r_{i}\right) \delta q_{i}
\end{aligned}
$$

In Equation (30) the expressions on the right hand side represent the virtual work of the internal forces $f_{i}$ and the external forces $F_{i}$ which can be rewritten as

$$
\begin{aligned}
& \left(f_{i}+F_{i}\right) \delta R_{b}+\left(M_{f}+M_{F}\right) \delta \phi+M_{m} \delta q_{i} \\
& =\left(f_{i}+F_{i}\right) \delta R_{b}+\left(R_{i / b} \times f_{i}+R_{i / b} \times F_{i}\right) \delta \phi \\
& \quad+\sum_{i=o}^{n}\left(R_{i}-r_{i}\right) \times f_{i} k_{i} \delta q_{i}+\sum_{i=o}^{n}\left(R_{i}-r_{i}\right) \times F_{i} k_{i} \delta q_{i}
\end{aligned}
$$

By taking the work effect of all bodies, the total virtual work can be then given as

$$
\begin{aligned}
& \delta W_{f}+\delta W_{F}+\delta W_{m} \\
& =\left(f_{i}+F_{i}\right) \delta R_{b}+\left(M_{f}+M_{F}\right) \delta \phi+M_{m} \delta q_{i}
\end{aligned}
$$

Recalling that the linear momentum $P_{i}=m_{i} \dot{R}_{i}=m_{i} V_{i}$, then the first term in the left hand side of Equations (30) can be expressed in term of linear momentum as

$$
m_{i} \ddot{R}_{i} \delta R_{b}=\frac{\mathrm{d} P_{i}}{\mathrm{~d} t} \delta R_{b}
$$

Introducing the angular momentum of the ith body about the base

$$
L_{i / b}=R_{i / b} \times m_{i} V_{i}
$$

Using the latter expression (34) and Equation (26), the middle and the last terms in the left hand side of Equation (30) can be rewritten respectively, as

$$
\begin{aligned}
m_{i} \ddot{R}_{i} \cdot \delta \phi \times R_{i / b} & =\delta \phi \cdot R_{i / b} \times m_{i} \ddot{R}_{i} \\
& =\left(\frac{\mathrm{d} L_{i / b}}{\mathrm{~d} t}-\dot{R}_{i / b} \times P\right) \delta \phi \\
m_{i} \ddot{R}_{i} \cdot \sum_{i=o}^{n} k_{i} \times\left(R_{i}-r_{i}\right) \delta q_{i} & =\sum_{i=o}^{n} k_{i} \cdot\left(R_{i}-r_{i}\right) \times m_{i} \ddot{R}_{i} \cdot \delta q_{i} \\
& =\left(\frac{\mathrm{d} L_{m}}{\mathrm{~d} t}-\left(\dot{R}_{i}-\dot{r}_{i}\right) \times P\right) \delta q_{i}
\end{aligned}
$$

where $L_{m}=\left(R_{i}-r_{i}\right) \times m_{i} V_{i}$.

After all, the virtual work of the whole system is presented as follows

$$
\begin{aligned}
& \frac{\mathrm{d} P}{\mathrm{~d} t} \delta R_{b}+\left(\frac{\mathrm{d} L_{i / b}}{\mathrm{~d} t}-\dot{R}_{\mathrm{i} / b} \times P\right) \delta \phi+\left(\frac{\mathrm{d} L_{m}}{\mathrm{~d} t}-\left(\dot{R}_{i}-\dot{r}_{i}\right) \times P\right) \delta q \\
& =(f+F) \delta R_{b}+\left(M_{f}+M_{F}\right) \delta \phi+M_{m} \delta q
\end{aligned}
$$

Since the virtual variations $\delta R_{b}, \delta \phi$ and $\delta q$ are independent we can reach the well known variation of linear and angular momentum equations, respectively, as

$$
\frac{\mathrm{d} P}{\mathrm{~d} t}=f+F
$$




$$
\begin{aligned}
& \left(\frac{\mathrm{d} L_{i / b}}{\mathrm{~d} t}-\dot{R}_{\mathrm{i} / \mathrm{b}} \times P\right)+\left(\frac{\mathrm{d} L_{m}}{\mathrm{~d} t}-\left(\dot{R}_{i}-\dot{r}_{i}\right) \times P\right) \\
& =M_{f}+M_{F}+M_{m}
\end{aligned}
$$

According to Equations (38) and (39), there are three conditions that the moments of forces about the virtual axis of rotation to vanish, that is

$$
\begin{gathered}
\frac{\mathrm{d} P}{\mathrm{~d} t}=0 \\
\frac{\mathrm{d} L_{\mathrm{i} / \mathrm{b}}}{\mathrm{d} t}-V_{i / b} \times P=0 \\
\frac{\mathrm{d} L_{m}}{\mathrm{~d} t}-\left(\dot{R}_{i}-\dot{r}_{i}\right) \times P=0
\end{gathered}
$$

Note that Equation (40) is guaranteed automatically by the law of conservation of linear momentum. To ensure the validity of (41) there are two requirements must be met, first

$$
\begin{gathered}
V_{i / b} \times P \delta \phi=V_{i / b} \times V_{c} \cdot m_{t} \delta \phi=0 \\
\left(\dot{R}_{i}-\dot{r}_{i}\right) \times P \delta q=\left(\dot{R}_{i}-\dot{r}_{i}\right) \times V_{c} \cdot m_{t} \delta q=0
\end{gathered}
$$

which requires that $V_{i / b}$ and $V_{c}$ are parallel, $\left(\dot{R}_{i}-\dot{r}_{i}\right)$ and $V_{c}$ are parallel, where $V_{c}$ is the velocity of center of mass of the robotic system. The second requirement is that $M_{F} \delta \phi=0$, that is the projection of $\mathrm{d} L_{i / b}$ onto the direction $\delta \phi$ is conserved, then

$$
\frac{\mathrm{d} L_{i / b}}{\mathrm{~d} t} \delta \phi=\frac{\mathrm{d}}{\mathrm{d} t}\left(L_{i / b} \hat{l}_{1}\right) \delta \phi=0
$$

where $\hat{l}_{1}$ is a unit vector along the virtual axis of rotation $Z$. From (45) it follows that

$$
L_{i / b} \hat{l}_{1}=\text { const }
$$

While the third condition can be guaranteed by $M_{m} \delta q$, that is the projection

$$
\frac{\mathrm{d} L_{m}}{\mathrm{~d} t} \delta q=\frac{\mathrm{d}}{\mathrm{d} t}\left(L_{m} \hat{l}_{2}\right) \delta q=0
$$

Similarly, it follows from (47) that

$$
L_{m} \hat{l}_{2}=\text { const }
$$

Theorem 1: A totally free-flying space robot defined by d'Almberts-Lagrange dynamics (5) is said to be nonholonmic system if conditions (23), (24), (26) and (28) are satisfied.

According to the previous analysis, when external forces exert no moment around the axis of rotation, the conservation of momentum holds. From geometrical view point, if the ith-body's relative linear velocity with respect to the base satellite is parallel to the linear velocity of the system center of mass, and if the relative linear velocity between the ith-body's centroid and its joint are parallel to the linear velocity of the system center of mass, and if the projections of angular momentum is along their corresponding angular displacements, then the system poses a nonholonomic constraints. In other words, any motion in base satellite or the manipulator or both will cause the system to adjust its motion to keep the direction of base linear velocity, and the projections of the angular momentum parallel to the virtual axis of rotation. It also embodies that the momentum is transferred from/to the manipulator to/from the base to maintain the momentum constant.

\subsection{Free-Flying Space Robot Interacting with a Target Satellite}

We assume now that the space robot established a contact with a target satellite. It is desired to find out the conditions to keep the momentum conserved. A similar analysis to part A above is followed.

Applying the principle of virtual work by d'Almberts-

$$
\sum_{i=0}^{n+1}\left(m_{i} \ddot{R}_{i}-f_{i}-F_{i}\right) \delta R_{i}=0
$$

and from (6) the virtual displacement of the target is given as

$$
\begin{aligned}
\delta R_{i}= & \delta R_{b}+\delta \phi \times R_{i / b}+\sum_{i=o}^{n} k_{i} \times\left(R_{i}-r_{i}\right) \delta q_{i} \\
& +\delta \phi_{T} \times R_{T / E E}+\delta r_{T}
\end{aligned}
$$

Substituting (50) and (29) into (49) yields into

$$
\begin{aligned}
& m_{i} \ddot{R}_{i} \cdot \delta R_{b}+m_{i} \ddot{R}_{i} \cdot \delta \phi \times R_{i / b}+m_{i} \ddot{R}_{i} \cdot \sum_{i=o}^{n} k_{i} \times\left(R_{i}-r_{i}\right) \delta q_{i} \\
& +m_{T} \ddot{R}_{T} \cdot \delta \phi_{T} \times R_{T / E E}+m_{T} \ddot{R}_{T} \cdot \delta r_{T}=f_{i} \cdot \delta R_{b}+f_{i} \cdot \delta \phi \times R_{i / b} \\
& +f_{i} \cdot \sum_{i=o}^{n} k_{i} \times\left(R_{i}-r_{i}\right) \delta q_{i}+F . \delta R_{b}+F_{i} \cdot \delta \phi \times R_{i / b} \\
& +F_{i} \cdot \sum_{i=o}^{n} k_{i} \times\left(R_{i}-r_{i}\right) \delta q_{i}+f_{T} \cdot \delta r_{T}+f_{i} \cdot \delta \phi_{T} \times R_{T T / E E} \\
& +F_{T} \cdot \delta r_{T}+F_{T} \cdot \delta \phi_{T} \times R_{T / E E}
\end{aligned}
$$

The last line in (51) can be expressed in terms of virtual work as

$$
\begin{aligned}
& \delta W_{f T}+\delta W_{F T}+\delta W_{T r}=f_{T} \cdot \delta r_{T}+f_{T} \cdot \delta \varphi_{T} \times R_{T / E E} \\
& +F_{T} \cdot \delta r_{T}+F_{T} \cdot \delta \varphi_{T} \times R_{T / E E}
\end{aligned}
$$

Rewriting the terms $m_{T} \ddot{R}_{T} \cdot \delta \varphi_{T} \times R_{T / E E}$ and $m_{T} \ddot{R}_{T} \cdot \delta \phi_{T}$ in (51), respectively, as

$$
\begin{gathered}
m_{T} \ddot{R}_{T} \delta r_{T}=\frac{\mathrm{d} P_{T}}{\mathrm{~d} t} \delta r_{T} \\
m_{T} \ddot{R}_{T} \cdot \delta \phi_{T} \times R_{T / E E}=\delta \phi_{T} \cdot R_{T / E E} \times m_{T} \ddot{R}_{T} \\
=\left(\frac{\mathrm{d} L_{T / E E}}{\mathrm{~d} t}-\dot{R}_{T / E E} \times P_{T}\right) \delta \phi_{T}
\end{gathered}
$$


where $L_{T / E E}=R_{T / E E} \times m_{T} V_{T}$.

After all, the virtual work of the whole system is presented as follows

$$
\begin{aligned}
& \frac{\mathrm{d} P}{\mathrm{~d} t} \delta R_{b}+\left(\frac{\mathrm{d} L_{i / b}}{\mathrm{~d} t}-\dot{R}_{\mathrm{i} / b} \times P\right) \delta \phi+\left(\frac{\mathrm{d} L_{m}}{\mathrm{~d} t}+\left(\dot{R}_{i}-\dot{r}_{i}\right) \times P\right) \delta q \\
& +\frac{\mathrm{d} P_{T}}{\mathrm{~d} t} \delta r_{T}+\left(\frac{\mathrm{d} L_{T / E E}}{\mathrm{~d} t}-\dot{R}_{T / E E} \times P_{T}\right) \delta \phi_{T} \\
& =(f+F) \delta R_{b}+\left(M_{f}+M_{F}\right) \delta \phi+M_{m} \delta q \\
& +\left(f_{T}+F_{T}\right) \cdot \delta r_{T}+\left(M_{f T}+M_{F T}\right) \delta \phi
\end{aligned}
$$

According to Equation (55), there are five conditions that the moments of forces about the virtual axis of rotation to vanish. Three of these conditions are similar to the conditions (40)-(42), in addition to

$$
\begin{gathered}
\frac{\mathrm{d} P_{T}}{\mathrm{~d} t}=0 \\
\left(\frac{\mathrm{d} L_{T / E E}}{\mathrm{~d} t}-\dot{R}_{T / E E} \times P_{T}\right)=0
\end{gathered}
$$

Condition (56) implies that $P_{T}=$ const , that is

$$
V_{T}=\text { const }
$$

Meanwhile, conditions (57) implies

$$
L_{T / E E} \hat{l}_{3}=\text { const }
$$

And

$$
\dot{R}_{T / E E} \times P_{T}=v_{T / E E} \times m_{T} V_{T}
$$

Theorem 2: A combined free-flying space robot interacting with a target satellite defined by d'AlmbertsLagrange dynamics (3.29) is said to nonholonmic system if conditions (3.23), (3.24), (3.26), (3.28) and (3.38)(3.40) are satisfied.

Then, in addition to the conditions concluded in the case of free-flying space robot, it is concluded also that to hold a constant momentum: 1) the target linear velocity should not change; 2 ) the linear relative velocity between the target and the end-effector should be in the same direction; 3) and its relative angular momentum projection should be kept constant. Figure 2 interprets these conditions.

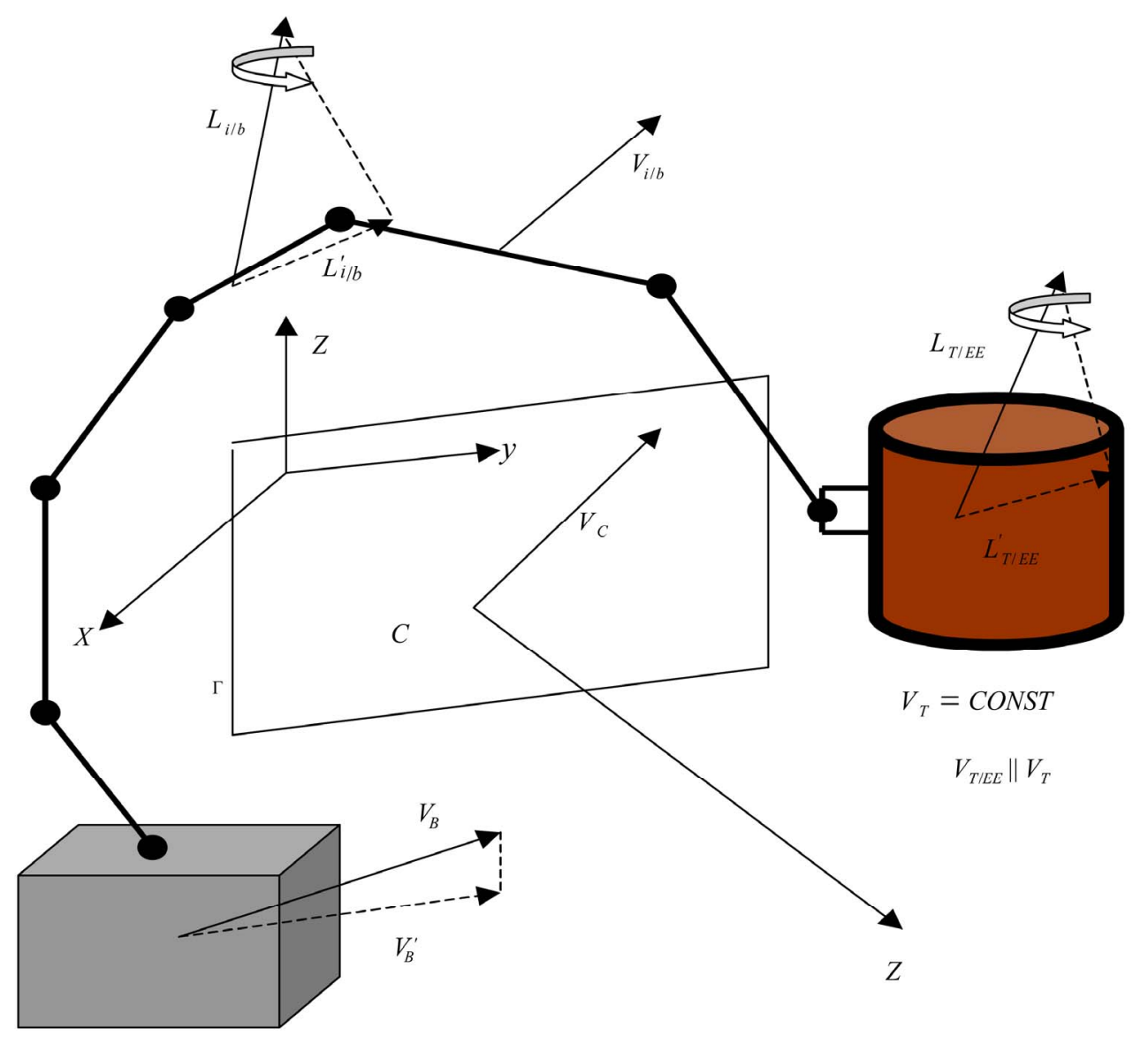

Figure 2. Nonholonomic conditions interpretation of a free-flying space robot interacting with a target satellite. 


\section{Nonholonomy Criterion with Zero-Initial Momentum Condition (Non-Drifted System)}

Holonomic kinematical conditions can be attacked in two approaches. If there are $m$ equations between $n$ variables, we can eliminate $m$ of these and reduce the problem to $N-m$ independent variables. However, this elimination may be rather cumbersome. Moreover, the conditions between the variables may be of a form that makes distinction between dependent and independent variables artificial. Another approach is to operate with surplus number of variables and retain the given constraint relations as auxiliary conditions. Nonholonomic conditions necessitate the second way of treatment. A reduction in variables is not possible here because the equations for eliminating some variables as dependent variables do not exist. Thus, we have to operate with more variables than degrees of freedom of the system demand.

Establishing criteria that determine whether a mechanical system is holonomic or nonholonomic is so crucial. In case of a mechanical system with linear kinematic kenimatic-like constraints, a necessary and sufficient condition is needed.

Using Lie algebra and bilinear covariants is cumbersome and time consuming in the case of complicated systems. In [12-13], another alternative sufficient and necessary condition is discussed by proposing a linear transformation to verify the integrability of holonomic systems. But in the latter approach, it is not clear how to construct the augmented matrix which is necessary to build the transformation matrix.

In this work, a transformation matrix is proposed to construct a linear transformation by using the concept of orthogonal projection techniques. This matrix is sufficient and necessary to verify the integrability of holonomic and nonholonomic system. Let us first define a system subjected to linear kinematic (or kienmatic-like) constraints given in the form

$$
A \dot{\theta}=0
$$

where the constriant matrix $A \in R^{m \times N}$ and the generalized variables $\dot{\theta} \in R^{N}$. For a system to be holonomic, a complete integrability of all $\mathrm{m}$ equations has to be fulfilled. But it might not be possible to find out whether a system of linear constraints are integrable because of unavailability of integrablity techniques or time consuming.

Geometrically, the constraint (61) mean that a point of an $N$-dimensional space $R\left(\theta_{1}, \theta_{2}, \cdots, \theta_{N}\right)$ cannot be displaced arbitrarily, but it must move a long a curve that touches at each of its points a hyperplane $L^{N-m}$ of di- mension $N-m$, which contains all displacement vectors $\mathrm{d} \theta_{1}, \mathrm{~d} \theta_{2}, \cdots, \mathrm{d} \theta_{N}$ satisfying the constraint Equation (61). A system of Pfaffian equations is completely integrable if all admissible curves emanating from any point in the space lie on a surface of dimension $N-m$ passing through that point. However, if the system is not integrable, any point in the configuration space can be reached, although its possible displacement is restriced.

Theorem 3: For a system subjected to linear kinematic (kinematic-like) constraint defined in (61), these constraint are said to be holonomic constraint if we can construct a linear trasformation matrix $T$ that maps all vectors lying in $T^{N-m}$ to zero and maps all vectors orthogonal to the hapersurface $L^{N-m}$ onto themselves.

Proof: A linear transformation matrix $T$ is introduced that is defined at each point of the space $R\left(\theta_{1}, \theta_{2}, \cdots, \theta_{N}\right)$. This transformation maps all vectors lying in $T^{N-m}$ to zero and maps all vectors orthogonal to the hapersurface $L^{N-m}$ onto themselves.

In other words, this transformation $T$ maps all displacement vectors $\mathrm{d} \theta_{1}, \mathrm{~d} \theta_{2}, \cdots, \mathrm{d} \theta_{N}$ satisfying the constraint (61) to the null vector as follows

$$
\dot{\Gamma}=T \dot{\theta}
$$

where $\dot{\Gamma} \in R^{m}$ and $T \in R^{m \times N}$.

Hence from (62) and the definition of the transformation $T$,

$$
T \dot{q}=\mathrm{Y} A \dot{\theta}
$$

where the matrix $Y \in R^{m \times m}$ and undetermined yet. Vectors orthogonal to $L^{N-m}$, in particular, the $m$ rows $A_{i}$ are mapped by the transformation $T$ onto themselves as

$$
\mathrm{YAA} A_{i}=T A_{i}=A_{j}
$$

The condition (64) admits a unique solution of $\mathrm{Y}$ and its elements should not vanish for a system to be holonomic.

To find the elements of the matrix $\mathrm{Y}$, we augment the matrix $A$, which is assumed to have full rank $m$, making it into a square matrix $A$ in such a way such that $\tilde{A} \in R^{N \times N}$ its determinant does not vanish. The elements $y_{i j}$ of $\mathrm{Y}$ can then be found uniquely and are the elements of first $m$ rows and first $m$ columns of the inverse augmented matrix $A$. But as it can be seen in [12], it is not clear how to augment the matrix $A$.

In this work, a holonomy scheme is proposed to construct the augmented matrix $A$ by using the orthogonal projectors. This scheme can easily be used to verify the holonomy of constrained systems. From the theory of linear algebra [18], the constrained system defined in (61) is equivalent to the following unconstrained but larger linear system 


$$
\left[\begin{array}{c}
A \\
P_{\perp}
\end{array}\right] \dot{q}=\left[\begin{array}{l}
0 \\
0
\end{array}\right]
$$

where $P_{\perp}$ is an orthogonal projector on the space $R^{m \times N}$. The projector $P_{\perp}$ can be found easily by the generalized Pseudoinverse and singular value decomposition techniques.

Using the Moore-Penrose inverse, the solution of (61) is

$$
\dot{q}=\left(I-A^{+} A\right) \dot{v}
$$

where $I \in R^{N \times N}$ is the identity matrix, the vector $\dot{v}$ is an arbitrary vector, and $A^{+}$is the Penrose inverse defined as $A^{+}=A^{T}\left(A A^{T}\right)^{-1}$. Let $D=\left(I-A^{+} A\right)$. Since (61) is a constrained system, the solution should be

$$
\dot{q}=P_{\perp} \dot{v}
$$

where $P_{\perp}$ is of $N \times(N-m)$ dimension. All the columns of the matrix $D$ are in the null space of $A$ that is $A D=0$. Then any $n-m$ linearly independent columns of $D$ can be chosen to form $P_{\perp}$. Now we use the singular value decomposition for $D$ to select the proper columns of $P_{\perp}$ such that $D=U \Sigma V^{T}$, where $U$ and $V^{T}$ are unitary orthogonal matrices of size $N \times N$ and the diagonal matrix $\Sigma$ with nonnegative diagonal elements in decreasing order as

$\Sigma=\operatorname{diag}\left\{\kappa_{1}, \kappa_{2}, \cdots, \kappa_{N-m}, 0, \cdots, 0\right\}$. Since $V^{T}$ is an orthogonal matrix, and $A D=A\left(U \Sigma V^{T}\right)=0$, the

$A U \Sigma=0$. Looking at the structure of $\Sigma$ it can be seen that the first $N-m$ columns of $U$ are in the null space of $A$. Then the first $N-m$ columns of $U$ can be used as the orthogonal projector $P_{\perp}$.

Now the extended augmented matrix $A$ can be defined as

$$
\tilde{A}=\left[\begin{array}{c}
A \\
P_{\perp}
\end{array}\right]
$$

which is of full rank. By finding the inverse of the augmented matrix $\tilde{A}$, then the unique matrix $\mathrm{Y}$ is composed of the elements of the first $m$ rows and first $m$ columns of the inverse augmented matrix $\tilde{A}$ as:

$$
\begin{gathered}
\tilde{A}^{-1}=\left[\begin{array}{cc}
\tilde{A}_{11}^{-1} & \tilde{A}_{12}^{-1} \\
\tilde{A}_{21}^{-1} & \tilde{A}_{22}^{-1}
\end{array}\right] \\
Y=\tilde{A}_{-11}^{1} \in R^{m \times m}
\end{gathered}
$$

By then, the transformation matrix $T$ can be constructed as $T=\mathrm{YA}$. By this end, a necessary and sufficient condition is obtained to verify the integrability of constrained system. If the transformation matrix $T$ is not rank deficient, then the system is integrable.

Note that as in (13) the system is subjected to a nonholonomic (non-integrable) constraint because of con- servation of angular momentum in the absence of external forces. The physical meaning behind these constraints is that they restrict the kinematically possible displacements (possible values of the velocities) of the individual parts of the system. On the contrast, the linear momentum results in a holonomic (integrable) constraint.

Note that in the case of linear momentum, the constraint equation

$$
A_{\text {linear }}=\left[\begin{array}{lllll}
M_{V_{b}} & M_{V_{b} \Omega_{b}} & M_{V_{b} q} & M_{V_{b} \omega_{T}} & M_{V_{b} v_{T}}
\end{array}\right]
$$

while for the angular momentum is given as

$$
A_{\text {angular }}=\left[\begin{array}{lllll}
M_{v_{b} \Omega_{b}}^{T} & M_{\Omega_{b}} & M_{\Omega_{b} q} & M_{\Omega_{b} \omega_{T}} & M_{\Omega_{b} v_{T}}
\end{array}\right]
$$

\section{Nonholonomy Criterion with Non-Zero Initial Conditions (Drifted Systems)}

Establishing criteria that determine whether a mechanical system is holonomic or nonholonomic is so crucial. In case of a mechanical system with linear kenimatic-like constraints, a necessary and sufficient condition is needed.

Using Lie algebra and bilinear covariants is cumbersome and time consuming in the case of complicated systems. In [11,12], another alternative sufficient and necessary condition is discussed by proposing a linear transformation to verify the integrability of holonomic systems. But in the latter approach, it is not clear how to construct the augmented matrix which is necessary to build the transformation matrix.

In this work, a transformation matrix is proposed to construct a linear transformation by using the concept of orthogonal projection techniques. This matrix is sufficient and necessary to verify the integrability of holonomic and nonholonomic system. Let us first define a system subjected to linear kinematic (or kienmatic-like) constraints given in the form

$$
A \dot{\theta}=c
$$

where the constraint matrix $A \in R^{m \times N}$ represents linear or angular momentum constraint matrix, and the generalized velocities $\dot{\theta} \in R^{N}$ as $\dot{\theta}=\left[\begin{array}{lllll}V_{b}^{T} & \Omega_{b}^{T} & \dot{q}^{T} & \omega_{T}^{T} & v_{T}^{T}\end{array}\right]$, and the vector $c$ represents the vector of momentum initial conditions.

The constraint (73) can be modified in away such that the time displacement is considered a long with other generalized variables as follows:

$$
\operatorname{Ad} \theta=c \mathrm{~d} t
$$

or

$$
\left[\begin{array}{ll}
A & -C
\end{array}\right]\left[\begin{array}{c}
\mathrm{d} \theta \\
\mathrm{d} t
\end{array}\right]=0
$$


Let $A_{1}=\left[\begin{array}{ll}A & -C\end{array}\right] \in R^{3 \times(N+1)}$ be defined as the modified constraint matrix and the displacement vector $\mathrm{d} \phi=\left[\begin{array}{c}\mathrm{d} \theta \\ \mathrm{d} t\end{array}\right] \in R^{N+1}$. In case of linear momentum is defined as

$$
A_{1}=\left[\begin{array}{llllll}
M_{V_{b}} & M_{V_{b} \Omega_{b}} & M_{V_{b} q} & M_{V_{b} \omega_{T}} & M_{V_{b} V_{T}} & -C
\end{array}\right]
$$

while in case of the angular momentum is given as

$$
A_{1}=\left[\begin{array}{llllll}
M_{v_{b} \Omega_{b}}^{T} & M_{\Omega_{b}} & M_{\Omega_{b} q} & M_{\Omega_{b} \omega_{T}} & M_{\Omega_{b} v_{T}} & -C
\end{array}\right]
$$

In case of a free-flying space robot or losing contact with the target, the constraint matrix is, then, reduced to $A_{1}=\left[\begin{array}{llll}M_{V_{b}} & M_{V_{b} \Omega_{b}} & M_{V_{b} q} & -C\end{array}\right]$ for linear momentum, and $A_{1}=\left[\begin{array}{llll}M_{V_{b} \Omega_{b}}^{T} & M_{\Omega_{b}} & M_{\Omega_{b} q} & -C\end{array}\right]$ for angular momentum where all terms related to the target satellite is set to zero (see $[4,9,22]$ for more details).

From linear algebra point of view, Equation (73) represents a system of $m$ linear equations with $d \phi$ being the vector of unknowns. Since the number of equations is less than that of unknowns, it has infinitely many solutions given by

$$
\mathrm{d} \phi=P_{\perp} \mathrm{d} z
$$

for an arbitrary $z(\varsigma)$ function of the parameter $\varsigma$ and the orthogonal projector $P_{\perp}$ is an $(N+1) \times(N+1-m)$ dimensional full rank matrix whose column space is in the null space of , i.e.,

$$
A_{1} P_{\perp}=0
$$

For a system to be holonomic, a complete integrability of all $\mathrm{m}$ equations has to be fulfilled. But it might not be possible to find out whether a system of linear constraints is integrable because of unavailability of integrablity techniques or time consuming.

Geometrically, the constraint (73) mean that a point of an $(N+1)$-dimensional space $R\left(\theta_{1}, \theta_{2}, \cdots, \theta_{N}, \theta_{N+1}\right)$ cannot be displaced arbitrarily, but it must move a long a curve that touches at each of its points a hyperplane $L^{N+1-m}$ of dimension $N+1-m$, which contains all displacement vectors $\mathrm{d} \theta_{1}, \mathrm{~d} \theta_{2}, \cdots, \mathrm{d} \theta_{N+1}$ satisfying the constraint Equation (73), where $\theta_{N+1}$ represents the variable time $t$. A system of Pfaffian equations is completely integrable if all admissible curves emanating from any point in the space lie on a surface of dimension $N+1-m$ passing through that point. However, if the system is not integrable, any point in the configuration space can be reached, although its possible displacement is restricted.

To construct a linear transformation by using the concept of orthogonal projection techniques, a linear transformation matrix $T$ is defined at each point of the space $R\left(\theta_{1}, \theta_{2}, \cdots, \theta_{N}, \theta_{N+1}\right)$, which should maps all vectors lying in $T^{N+1-m}$ to zero and maps all vectors orthogonal to the hypersurface $L^{N+1-m}$ onto themselves.

For this purpose, the transformation $T$ is decomposed in such a way that,

$$
T=\mathrm{YA}_{1}
$$

where the matrix $Y \in R^{m \times m}$ of full rank and will be determined later. To obtain such a transformation, it is required that the $m$ rows $A_{\text {, }}$ are mapped by the transformation $T$ onto themselves as

$$
\mathrm{YAA} A_{i}=T A_{i}=A_{i}
$$

The condition (81) admits a unique solution of $\mathrm{Y}$ and its elements should not vanish for a system to be holonomic. To find the elements of the matrix $\mathrm{Y}$, we augment the matrix $A$ into a square matrix $\tilde{A} \in R^{(N+1) \times(N+1)}$ in such a way that its determinant does not vanish. The elements $y_{i j}$ of $\mathrm{Y}$ are the elements of first $m$ rows and first $m$ columns of the inverse augmented matrix.

From the theory of linear algebra, the constrained system defined in (73) is equivalent to the following unconstrained but larger linear system $[23,24]$ :

$$
\left[\begin{array}{l}
A_{1} \\
P_{\perp}
\end{array}\right] \mathrm{d} \phi=\left[\begin{array}{l}
0 \\
0
\end{array}\right]
$$

where the augmented matrix $A$ defined as

$$
\tilde{A}=\left[\begin{array}{l}
A_{1} \\
P_{\perp}
\end{array}\right]
$$

and $P_{\perp}$ is defined in (78).

In order to obtain $P_{\perp}$, treating $\dot{\theta}$ as the vector of unknowns, (25) can be solved using the Moore-Penrose inverse as

$$
\mathrm{d} \phi=\left(I-A_{1}^{+} A_{1}\right) \mathrm{d} v
$$

where $I \in R^{(N+1) \times(N+1)}$ is the identity matrix, the vector $\mathrm{d} v$ is an arbitrary vector, and $A_{1}^{+}$is the Moore-Penrose inverse defined as

$$
A_{1}^{+}=A_{1}^{T}\left(A_{1} A_{1}^{T}\right)^{-1}
$$

Equation (84) is similar to (78), but not exactly the same, as seen below.

Let $D=\left(I-A_{1}^{+} A_{1}\right)$. However, $D$ in (84) is not yet $P_{\perp}$ in (78) since they are of different dimensions. The projector $P_{\perp}$ is $(N+1) \times(N+1-m)$, whereas $D$ is an $(N+1) \times(N+1)$ square matrix. $P_{\perp}$ is of full rank but $D$ is not. Notice that we used $\mathrm{d} z(\varsigma)$ in (78) instead of $\mathrm{d} v(\varsigma)$ in (84). The rank of $D$ is $(N+3-m)$. All the columns of the matrix $D$ are in the null space of $A$, that is, $A D=0$. Then any $N+1-m$ linearly independent columns of $D$ can be chosen to form $P_{\perp}$. 
But it may create discontinuity in $\mathrm{d} v(\varsigma)$ if different set of linearly independent columns are chosen. To remedy this problem, we compute the singular value decomposition of $D$ to select the proper columns of $P_{\perp}$ such that

$$
D=U \Sigma V^{T}
$$

where $U$ and $V^{T}$ are orthogonal matrices of size $(N+1) \times(N+1)$, and the diagonal matrix $\Sigma$ with nonnegative diagonal elements in a decreasing order as

$$
\Sigma=\operatorname{diag}\left\{\kappa_{1}, \kappa_{2}, \cdots, \kappa_{N+1-m}, 0, \cdots, 0\right\}
$$

Let $u_{1}, u_{2}, \cdots, u_{N+3}$ denote the column vectors of $U$

$$
U=\left[\begin{array}{llll}
u_{1} & u_{2} & \cdots & u_{N+1}
\end{array}\right]
$$

Since $A D=A\left(U \Sigma V^{T}\right)=0$ and the matrix $V^{T}$ is orthogonal, then, $A U \Sigma=0$. Because the structure of $\Sigma$, it follows that the first $N+1-m$ columns of $U$ are in the null space of $A$, i.e.,

$$
A_{1} u_{i}=0, i=1,2, \cdots, N+1
$$

Then the first $N+1-m$ columns of $U$ may be chosen form the orthogonal projector $P_{\perp}$ as

$$
P_{\perp}=\left[\begin{array}{llll}
u_{1} & u_{2} & \cdots & u_{N+1-m}
\end{array}\right]
$$

It is obvious that $P_{\perp}$ is of full rank because $U$ is orthogonal.

Back to he extended augmented matrix $\tilde{A}$ defined in (83). By finding its inverse, then the unique matrix $\mathrm{Y}$ is composed of the elements of the first $m$ rows and first $m$ columns of the inverse augmented matrix $\tilde{A}$ as:

$$
\begin{gathered}
\tilde{A}_{1}^{-1}=\left[\begin{array}{cc}
\tilde{A}_{1}^{-1} & \tilde{A}_{1}^{-1} \\
\tilde{A}_{1}^{-1} & \tilde{A}_{1}^{-1}
\end{array}\right] \\
Y=\tilde{A}_{11}^{-1} \in R^{m \times m}
\end{gathered}
$$

By then, the transformation matrix $T$ can be constructed as $T=Y A_{1}$. By this end, a necessary and sufficient condition is obtained to verify the integrability of constrained system. If the transformation matrix $\mathrm{Y}$ is not rank deficient, then the system is integrable. The above can be summarized as follows.

Theorem 4: For a system subjected to linear kinematic (kinematic-like) constraint with nonzero initial conditions (momentum with a drift) defined in (73), these constraints are said to be holonomic constraint if we can construct a linear transformation matrix $T$ that maps all vectors lying in $T^{N+1-m}$ to zero and maps all vectors orthogonal to the hypersurface $L^{N+1-m}$ onto themselves.

\section{Simulation Results}

\subsection{Part 1 (Conservation of Momentum)}

A 6 DOF free-flying space robot is tested to verify its nonholonomic and holonomic behavior. The base satellite mass is assumed as $300 \mathrm{~kg}$, and eack of the 6 link mass is taken as $10 \mathrm{~kg}$. Simulation is run to plot the system momentum response assuming zeros external forces and zero initial conditions. The Figures $\mathbf{3}$ and $\mathbf{4}$ show that linear and angular momentum of a free-flying space robot is conserved and kept zero (range of 10e - 8). These results comply with the concept that for a free-flying

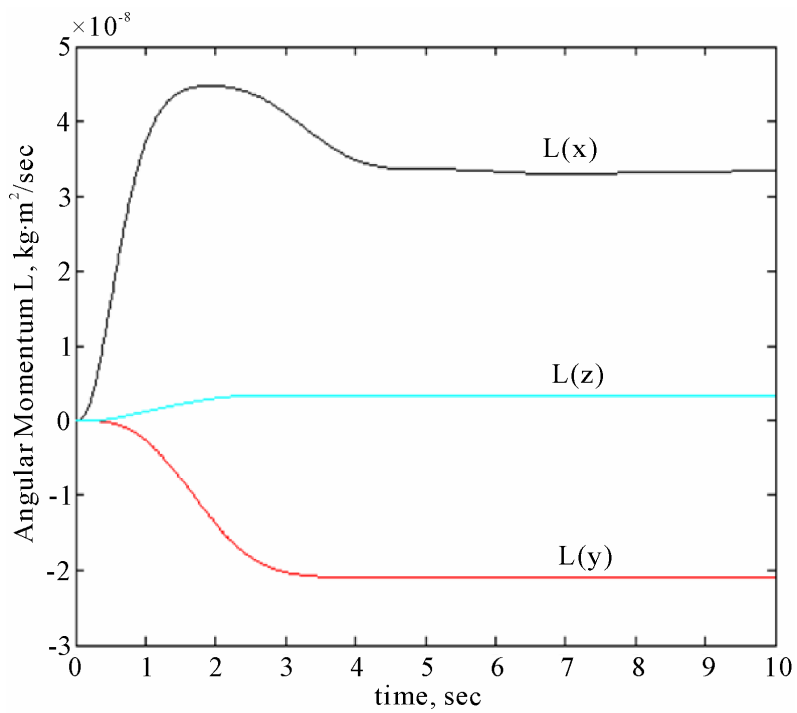

Figure 3. Angular momentum of a free-flying space robot.

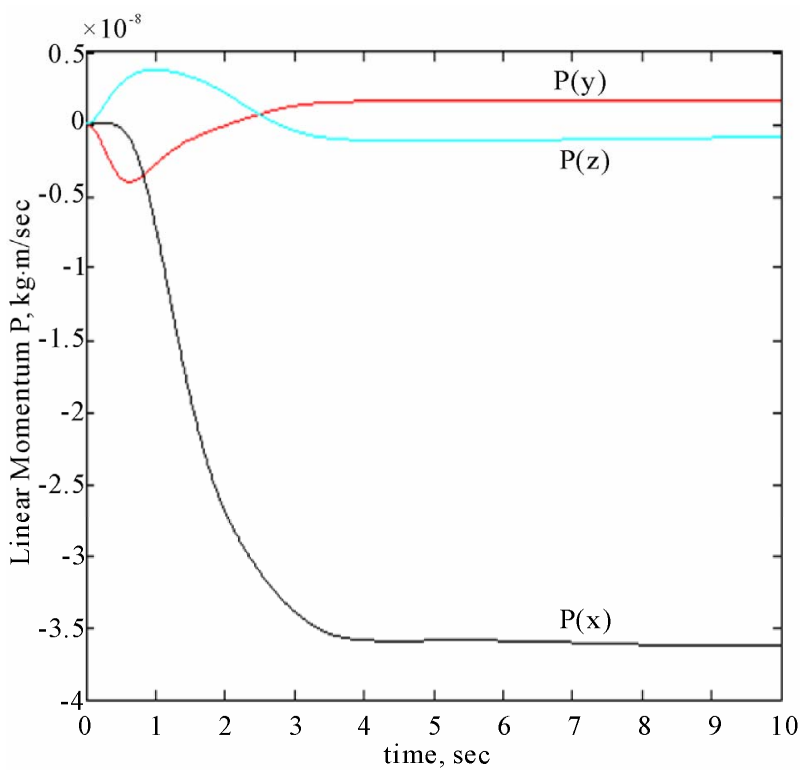

Figure 4. Linear momentum of a free-flying space robot. 
space robot its momentum is conserved in the absence of any external forces. On the other hand, Figures $\mathbf{5}$ and $\mathbf{6}$ show respectively non-conservation of linear and angular momentum of a free-flying space robot subjected to an external force.

Another simulation is also implemented to check the conservation of momentum of a free-flying space robot interacting with a target satellite as a combined system with nonzero initial linear velocity. The target satellite mass is assumed $1500 \mathrm{~kg}$. Figures 7 and 8 show that linear and angular momentum of a free-flying space together with its target is conserved and but since the initial conditions are assumed $10 \mathrm{~m} / \mathrm{sec}$ the momentum is hold at values different than zero. Figures $\mathbf{9}$ and $\mathbf{1 0}$ show non-conservation of momentum in case the target satel-

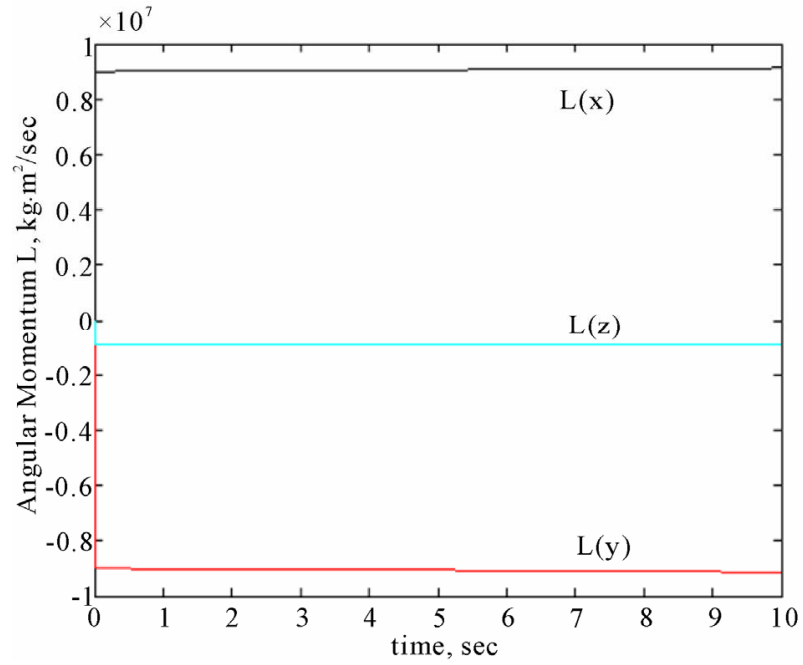

Figure 5. Angular momentum of a free-floating space robot in contact with a target satellite.

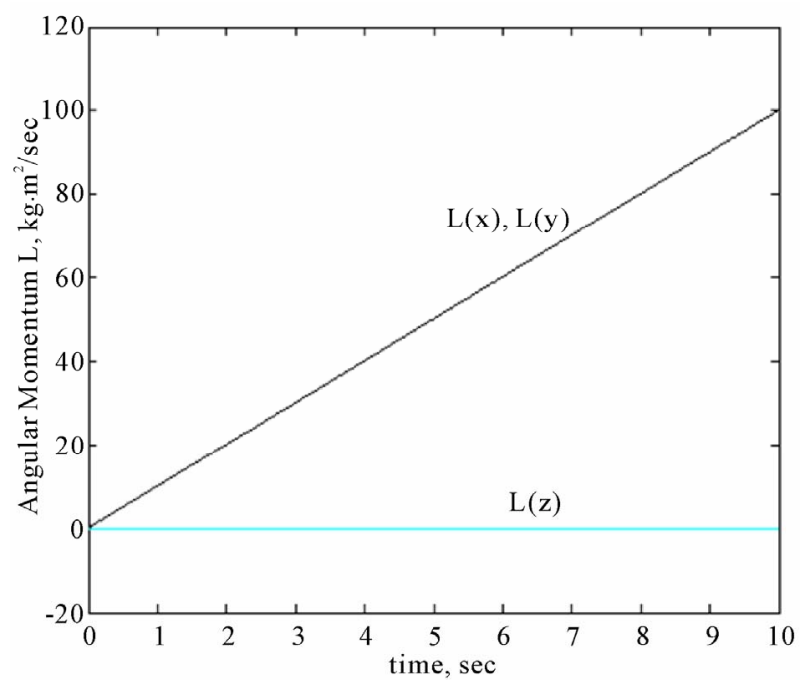

Figure 6. Angular momentum of a free-flying space robot subjected to an external force.

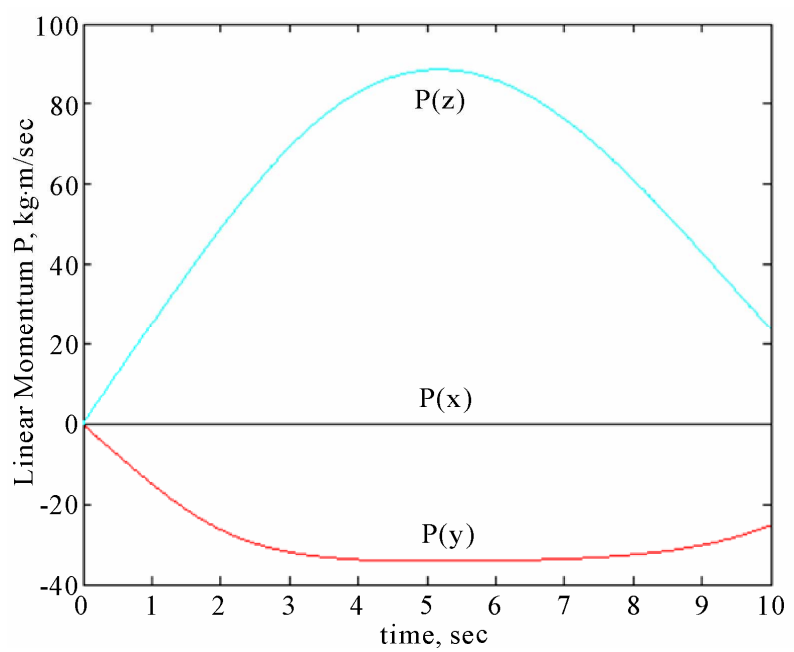

Figure 7. Linear momentum of a free-flying space robot subjected to an external force.

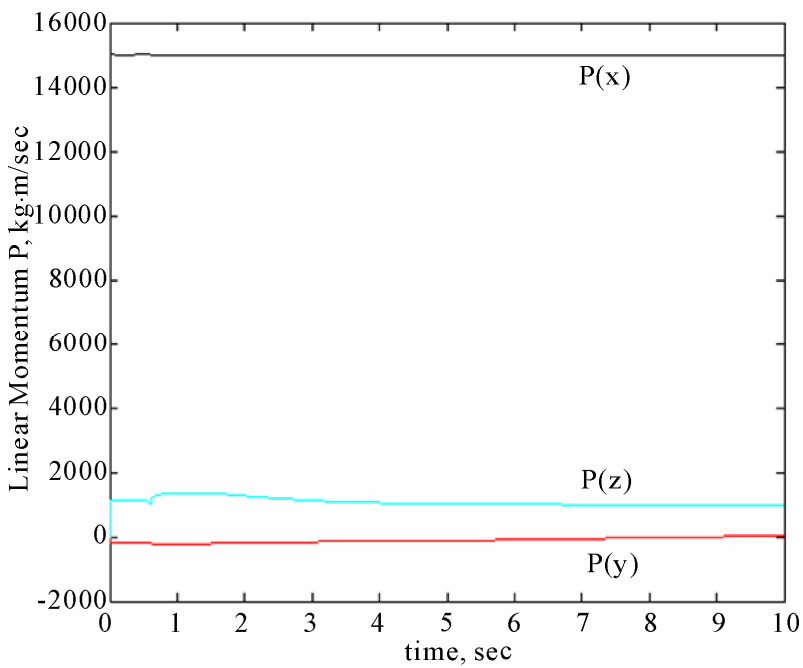

Figure 8. Linear momentum of a free-floating space robot in contact with a target satellite.

lite is changing and chosen for simulation as $1-\cos t$ in the $\mathrm{x}$-direction and this agrees with condition (58). Meanwhile, Figures 11 and $\mathbf{1 2}$ demonstrates the case when the end-effector moves in a direction not parallel to that of the target chosen $20 \mathrm{~m} / \mathrm{esc}$ in $\mathrm{x}$ and $\mathrm{y}$-direction and $20 \mathrm{~m} / \mathrm{sec}$ for the base satellite in $\mathrm{x}$-direction only and because it violates condition (60).

\subsection{Part 2 (Holonomy Matrix)}

A 6 DOF free-flying space robot is tested to verify its nonholonomic and holonomic behavior with zero initial linear velocity. The mass of the base satellite is assumed as $300 \mathrm{~kg}$, and the mass of each of the 6 links is taken as $10 \mathrm{~kg}$. The simulation results shows that and by using this algorithm the rank of the transformation matrix $T$ 


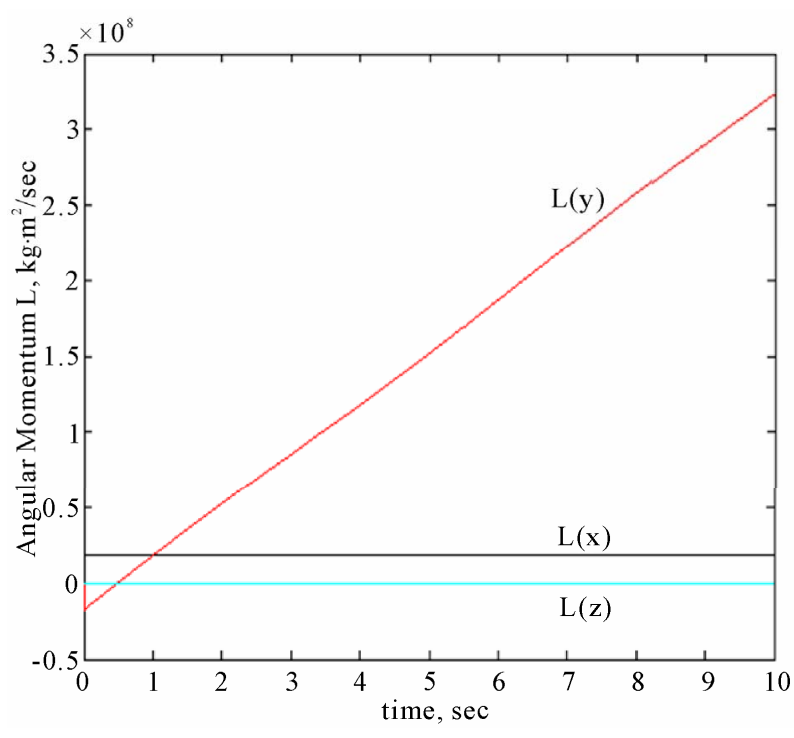

Figure 9. Angular momentum of a free-floating space robot in contact with a target satellite (violation of condition (58)).

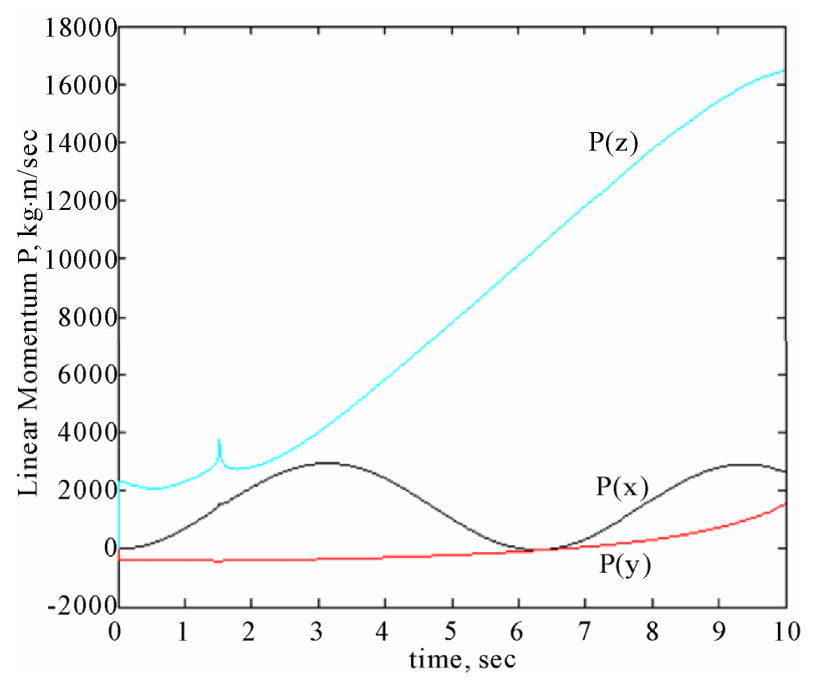

Figure 10. Linear momentum of a free-floating space robot in contact with a target satellite (violation of condition (58)).

is full $($ rank $=3)$ in the case of linear momentum while in the case of angular momentum the rank of the transformation matrix $T$ is not full rank (rank $=2$ ) as shown in Table 1. These results comply with the theoretical and physical results. Another simulation was run assuming external forces exposed to the space robot hand. It shows that the transformation matrix $T$ is of full rank ( $\mathrm{rank}=$ 3 ) in both cases of linear and angular momentum.

A 6 DOF free-flying space robot is tested to verify its nonholonomic and holonomic behavior with zero initial linear velocity. The mass of the base satellite is assumed as $300 \mathrm{~kg}$, and the mass of each of the 6 links is taken as $10 \mathrm{~kg}$. The initial momentum conditions are assumed as $\left[\begin{array}{lll}10 & 0 & 0\end{array}\right]$ for both linear and angular monetum. The simu-

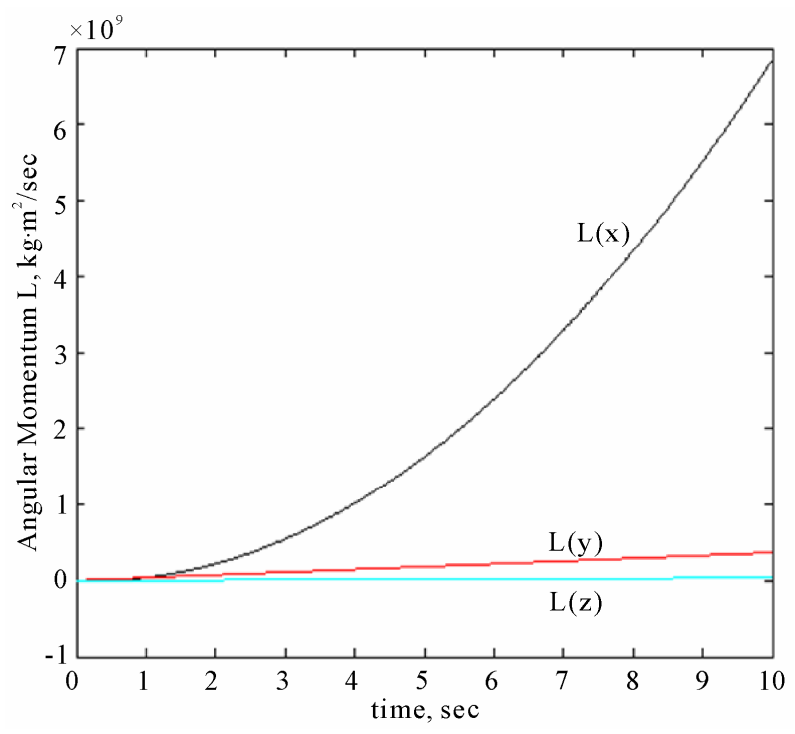

Figure 11. Angular momentum of a free-floating space robot in contact with a target satellite (violation of condition (60)).

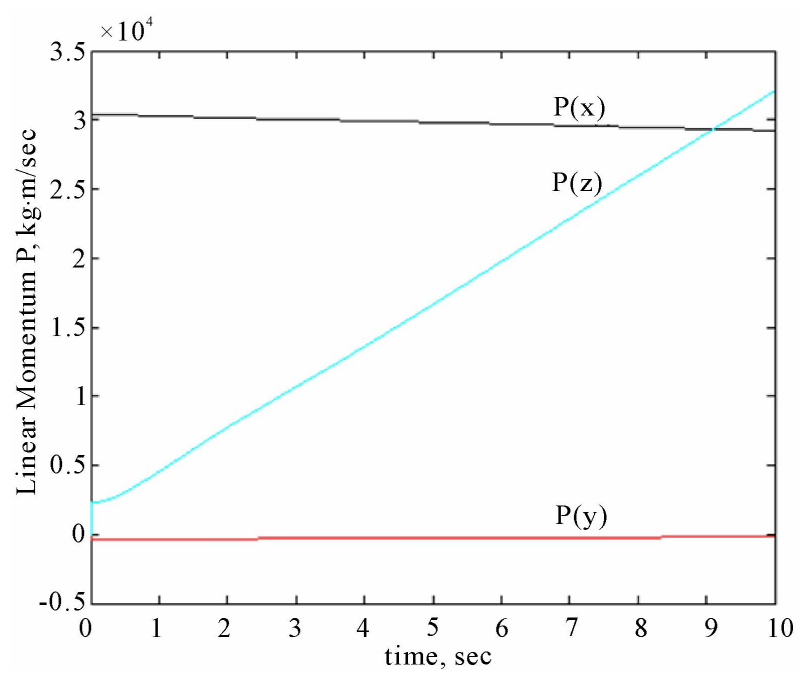

Figure 12. Linear momentum of a free-floating space robot in contact with a target satellite (violation of condition (60)).

lation results shows that and by using this algorithm the rank of the transformation matrix $\mathrm{Y}$ is full (rank=3) in the case of linear momentum while in the case of angular momentum the rank of the transformation matrix $\mathrm{Y}$ is not full rank (rank = 2) (see Table 2). These results comply with the theoretical and physical results. Another simulation was run assuming external forces exposed to the space robot end-effector. It shows that the transformation matrix $\mathrm{Y}$ is of full rank (rank = 3) in both cases of linear and angular momentum.

This approach is also implemented to check the holonomy of a space robot interacting with a target satellite as a combined system with nonzero initial linear velocity $20 \mathrm{~m} / \mathrm{sec}$ for both the base satellite and the target 
Table 1. Rank of the transformation matrix Y (sample).

\begin{tabular}{|c|c|c|}
\hline Case & Y matrix & Rank \\
\hline $\begin{array}{l}\text { Angular momentum of a free-flying space } \\
\text { robot with no external forces }\end{array}$ & 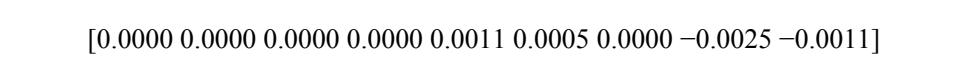 & 2 \\
\hline $\begin{array}{l}\text { Angular momentum of a free-flying space } \\
\text { robot with external forces }(10 \mathrm{~N})\end{array}$ & 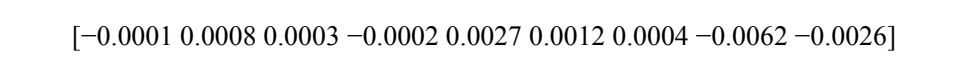 & 3 \\
\hline $\begin{array}{l}\text { Linear momentum of a free-flying space robot } \\
\text { with no external forces }\end{array}$ & 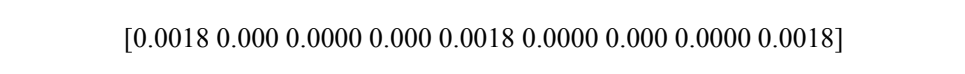 & 3 \\
\hline $\begin{array}{l}\text { Linear momentum of a free-flying space robot } \\
\text { with external forces }(10 \mathrm{~N})\end{array}$ & 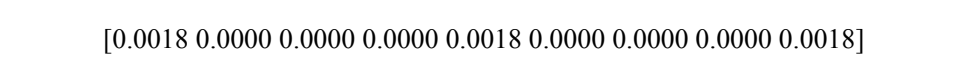 & 3 \\
\hline $\begin{array}{l}\text { Angular momentum of a free-flying space } \\
\text { robot interacting with a target satellite }\end{array}$ & 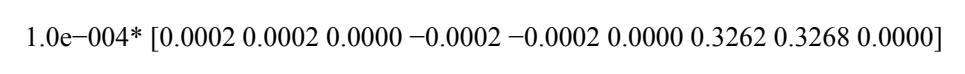 & 2 \\
\hline $\begin{array}{l}\text { linear momentum of a free-flying space robot } \\
\text { interacting with a target satellite }\end{array}$ & 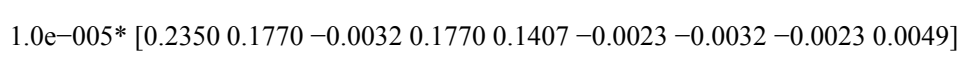 & 3 \\
\hline
\end{tabular}

Table 2. Rank of the nonholonomy matrix Y (sample).

\begin{tabular}{|c|c|c|}
\hline Case & Y matrix & Rank \\
\hline $\begin{array}{l}\text { Angular momentum of a free-flying space } \\
\text { robot with no external forces }\end{array}$ & {$[0.0000-0.0010-0.00040 .00000 .00260 .00140 .0000-0.0060-0.0026]$} & 2 \\
\hline $\begin{array}{l}\text { Angular momentum of a free-flying space } \\
\text { robot with external forces }(100 \mathrm{~N})\end{array}$ & 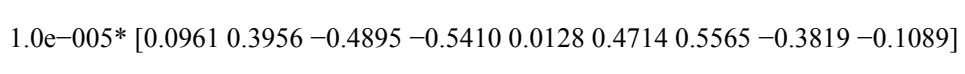 & 3 \\
\hline $\begin{array}{l}\text { Linear momentum of a free-flying space robot } \\
\text { with no external forces }\end{array}$ & 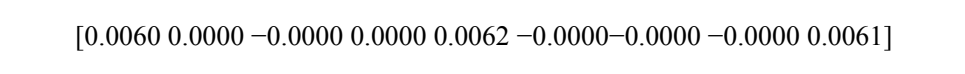 & 3 \\
\hline $\begin{array}{l}\text { Linear momentum of a free-flying space robot } \\
\text { with external forces }(100 \mathrm{~N})\end{array}$ & {$\left[\begin{array}{lllllllll}0.0022 & 0.0017 & 0.0016 & 0.0017 & 0.0033 & 0.0018 & 0.0016 & 0.0018 & 0.0025\end{array}\right]$} & 3 \\
\hline $\begin{array}{l}\text { Angular momentum of a free-flying space } \\
\text { robot interacting with a target satellite }\end{array}$ & $1.0 \mathrm{e}-004 *[-0.0000-0.00000 .00000 .00010 .0001-0.00010 .10940 .1104-0.0001]$ & 2 \\
\hline $\begin{array}{l}\text { linear momentum of a free-flying space robot } \\
\text { interacting with a target satellite }\end{array}$ & 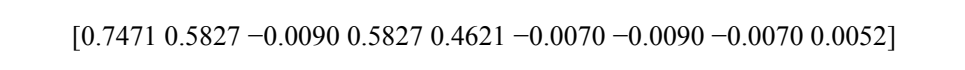 & 3 \\
\hline
\end{tabular}

satellite. The simulation shows that the holonomy matrix $\mathrm{Y}$ has full rank in case of the linear momentum, but it is rank-deficient in case of angular momentum. Which agrees with the theoretical approach considered in this approach that both linear and angular momentum are conserved but the linear momentum is holonomic and the angular momentum is nonholonomic.

\section{Conclusions}

From geometrical view point and in case of a totally free-flying space robot, if the linear velocity of the base satellite is parallel to the linear velocity of the system center of mass then it poses a holonomic constraint, and if the projections of angular momentum is along their corresponding angular displacements, then the system poses a nonholonomic constraints. In case of interacting with a un-actuated floating target satellite, another three conditions should be hold to keep the momentum constant. They are, the target linear velocity is constant, the relative linear velocity between the robot end-effector and the satellite should be parallel to that of the target satellite, and finally the angular momentum projection along the axis of rotation is hold constant as well.

For a system subjected to linear kinematic (kinematic-like) constraints, these constraint are said to be holonomic constraint the rank of the proposed linear transformation matrix is full, otherwise, it is said to be nonholonomic. This approach is useful to verify the non-violation of constraints in real-time application and to switch controllers or correct the situation.

\section{References}

[1] P. C. Hughes, "Spacecraft Attitude Dynamics," Wiley, New York, 1986.

[2] Z. Vafa and S. Dubbowsky, "The Kinematics and Dynamics of Space Manipulators: The Virtual Manipulator Approach," International Journal of Robotics Research, Vol. 9, No. 4, 1990, pp. 3-21. doi:10.1177/027836499000900401

[3] S. Dubowsky and E. Papadopoulos, "The Kinematic, Dynamics, and Control of Free-Flying and Free-Flaoting Space Robotic Systems," IEEE Transactions on Robotics and Automation, Vol. 9, No. 5, 1993, pp. 531-543. doi: $10.1109 / 70.258046$

[4] S. K. Saha, "A Unified Approach to Space Robot Kine- 
matics," IEEE Transactions on Robotics and Automation, Vol. 12, No. 3, 1996, pp. 401-405.

[5] Y. Umetani and K. Yoshida, "Resolved Motion Rate Control of Space Manipulators with Generalized Jacobian Matrix," IEEE Transactions on Robotics and Automation, Vol. 5, No. 3, 1989, pp. 303-314.

[6] X.-S. Ge, H. Li and Q.-Z. Zhang, "Nonholonomic Motion Planning of Space Robotics Based on the Genetic Algorithm with Wavelet Approximation," IEEE International Conference on Control and Automation, Guangzhou, 30 May-1 June 2007, pp. 1977-1980.

[7] D. R. Isenberg, "Dynamics and Simulation of a Space Robot in a Gravitational Field," IEEE Southeastcon, Huntsville, 3-6 April 2008, pp. 268-273.

[8] Y. Nakamura and R. Mukherjee, "Nonholonmic Path Planning of Space Robots via Bi-Directional Approach," IEEE Transactions on Robotics and Automation, Vol. 7, No. 4, 1991, pp. 500-514.

[9] T. Yoshikawa, "Dynamics Hybrid Position/Force Control of Robot Manipulators-Description of Hand Constraints and Calculation of Joint Driving Force," IEEE Journal of Robotics and Automation, Vol. 3, No. 5, 1987, pp. 386-392. doi:10.1109/JRA.1987.1087120

[10] Y. Xu and T. Kanade, "Space Robotics: Dynamics and Control," Kluwer Academic Publisher, Boston, 1993.

[11] A. De Luca and G. Oriolo, "Modeling and Control of Nonholonomic Mechanical Systems," In: J. Angeles and A. Kecskemethy, Eds., Kinematics and Dynamics of MultiBody Systems, CISM Courses and Lectures, Vol. 360, Springer-Verlag, Wien, 1995, pp. 277-342.

[12] Ju I. Neimark annd N. A. Fufaef, "Dynamics of Nonholonomic Systems," Vol. 33, Translations of Mathematical Monographs, American Mathematical Society, Providence, 1972.

[13] A. M. Lopsec, "Nichthholomome Systeme in Mehrdimensionalen Euklidischen Raumen," Trudy Seminara po Vektornomu i Tenzornomu Analizu, Vol. 4, 1937, pp. 302-317.

[14] A. M Bloch, M. Reyhanoglu and N. H. McClamroch, "Control and Stabilization of Nonholonomic Dynamic Systems," IEEE Transactions on Automatic Control, Vol.
37, No. 11, 1992, pp. 1746-1757. doi:10.1109/9.173144

[15] J. G. Wang, R. Mukherji, M. Ficocelli, A. Ogilvie, M. Liu and C. Rice, "Modeling and Simulation of Robotic System for Servicing Hubble Space Telescope," IEEE/RSJ International Conference on Intelligent Robots and Systems, Beijing, October 2006, pp. 1026-1031.

[16] F. Matsuno and K. Saito, "Attitude Control of a Space Robot with Initial Angular Momentum," IEEE International Conference on Robotics and Automation (ICRA), Vol. 2, 2001, pp. 1400-1440.

[17] H. T. Shui, J. W. Wang and H. X. Ma, "Optimal Motion Planning for Free-Floating Space Robots Based on Null Space Approach," International Conference on Measuring Technology and Mechatronics Automation, Zhangjiajie, 11-12 April 2009, pp. 845-848.

[18] C. Lanczos, "The Variational Principle of Mechanics," University of Toronto Press, Toronto, 1966.

[19] R. Murray and S. Sastry, "Nonholonomic Motion Planning: Steering Using Sinusoids," IEEE Transactions on Automatic Control, Vol. 38, No. 5, 1993, pp. 700-716.

[20] M. Shibli, "Unified Modeling Approach of Kinematics, Dynamics, and Control of a Free-Flying Space Robot Interacting with a Target Satellite," Journal of Intelligent Control and Automation, Vol. 2, No. 1, 2011, pp. 8-23.

[21] M. Shibli, "Modeling and Control of a Free-Flying Space Robot Interacting with a Target Satellite," PhD Thesis, Montreal, 2009.

[22] M. Shibli, F. Aghili and C.-Y. Su, "Modeling of a Free-Flying Space Robot in Contact with a Target Satellite," IEEE Conference on Control Applications, Toronto, 28-31 August 2005, pp. 559-564.

[23] A. Ben-Israel and N. E. T Greville, "Generalized Inverses: Theory and Application," 2nd Edition, Springer, New York, 2003.

[24] M. Shibli, C.-Y. Su and F. Aghili, "Online Nonholonomy Criterion of a Free-Flying Space Robot with/without Interaction with a Target Satellite," 36th International Symposium on Robotics, IEEE Robotics and Automation, Tokyo, 28 November-1 December, 2005. 


\section{Appendix A}

\section{Derivation of the Linear Velocity of a Target Satellite}

Referring to Figure 1, the position vector of the ith body with respect to the inertial frame can be readily expressed as

$$
r_{i}=r_{b}+r_{i / b}
$$

Moreover, the relative vector $R_{i / b}$ can be expressed in the form

$$
r_{i / b}=r_{i}-r_{b}
$$

Now the purpose is to derive the equation of that describes the linear velocity of the target satellite. From the principles of dynamics and because the target satellite is linked to the base satellite space servicing robot by the robot end-effector there is a relative linear and angular motion between the target satellite and the space robot.

The position of the contact point on the target satellite with respect to the base can be described based on (A1) as follows

$$
r_{t}=r_{b}+r_{t / b}
$$

Then the velocity of the contact point is determined by taking the time derivative of (A3), which yields

$$
V_{t}=V_{b}+\frac{\mathrm{d} r_{t / b}}{\mathrm{~d} t}
$$

The last term in (4) is evaluated as follows:

$$
\begin{aligned}
\frac{\mathrm{d} r_{t / b}}{\mathrm{~d} t} & =\frac{\mathrm{d}}{\mathrm{d} t}\left(x_{t} \hat{i}+y_{t} \hat{j}\right) \\
& =\frac{\mathrm{d} x_{t}}{\mathrm{~d} t} \hat{i}+x_{t} \frac{\mathrm{d} \hat{i}}{\mathrm{~d} t}+\frac{\mathrm{d} y_{t}}{\mathrm{~d} t} \hat{j}+y_{t} \frac{\mathrm{d} \hat{j}}{\mathrm{~d} t} \\
& =\left(\frac{\mathrm{d} x_{t}}{\mathrm{~d} t} \hat{i}+\frac{\mathrm{d} y_{t}}{\mathrm{~d} t} \hat{j}\right)+\left(x_{t} \frac{\mathrm{d} \hat{i}}{\mathrm{~d} t}+y_{t} \frac{\mathrm{d} \hat{j}}{\mathrm{~d} t}\right)
\end{aligned}
$$

\section{Appendix B}

\section{Linear and Angular Jacobian Matrices for a 6-DOF Based Satellite Space Robot Interacting with a Target Satellite}

Viewing the axes in three dimension, and noting that $\Omega_{b}=\Omega_{b} \hat{k}$, we can express the derivative (A6) and (A7) in terms of the cross product as

$$
\begin{aligned}
& \frac{\mathrm{d} \hat{i}}{\mathrm{~d} t}==\Omega_{b}: \hat{i} \\
& \frac{\mathrm{d} \hat{j}}{\mathrm{~d} t}==\Omega_{b}: \hat{j}
\end{aligned}
$$

Substituting these results into (A5) and using the distributive property of the vector cross product, one obtains

$$
\frac{\mathrm{d} r_{t / b}}{\mathrm{~d} t}=v_{t}+\Omega_{b}:\left(x_{t} \hat{i}+y_{t} \hat{j}\right)=v_{t}+\Omega: r_{t / b}
$$

Since the target satellite is linked with the space robot via the end-effector joint and using the Jacobian transformation, hence equation (A4) becomes

$$
V_{t}=V_{b}+\Omega_{b} \times r_{t_{/ b}}+J_{L_{E E}} \dot{q}+\omega_{t} \times r_{t_{/ E E}}+v_{t}
$$

The first three terms in the first parenthesis represent ured by an observer attached to the moving base coordinate system. This term will be denoted by $v_{t}$. The other three terms in the second parenthesis represent the instanenous time rate of change of the unit vector $\hat{i}, \hat{j}$ and $\hat{k}$ and measured in the inertial frame and given as:

$$
\begin{gathered}
\frac{\mathrm{d} \hat{i}}{\mathrm{~d} t}=\frac{\mathrm{d} \theta}{\mathrm{d} t}(\hat{j})=\Omega_{b} \hat{j} \\
\frac{\mathrm{d} \hat{j}}{\mathrm{~d} t}=\frac{\mathrm{d} \theta}{\mathrm{d} t}(-\hat{i})=-\Omega_{b} \hat{i}
\end{gathered}
$$

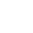




$$
\begin{gathered}
J_{A_{1}}=\left[k_{1}, 0,0,0,0,0,0\right] \\
J_{A_{2}}=\left[k_{1}, k_{2}, 0,0,0,0,0\right] \\
J_{A_{3}}=\left[k_{1}, k_{2}, k_{3}, 0,0,0,0\right] \\
J_{A_{4}}=\left[k_{1}, k_{2}, k_{3}, k_{4}, 0,0,0\right]
\end{gathered}
$$$$
J_{A_{5}}=\left[k_{1}, k_{2}, k_{3}, k_{4}, k_{5}, 0,0\right]
$$$$
J_{A_{6}}=\left[k_{1}, k_{2}, k_{3}, k_{4}, k_{5}, k_{6}, 0\right]
$$$$
J_{\mathrm{A}_{t}}=\left[k_{1}, k_{2}, k_{3}, k_{4}, k_{5}, k_{6}, k_{t}\right]
$$ 